\title{
Advanced Materials and Processes for High Energy Resolution Room Temperature Gamma Ray Spectrometers
}

PI: Douglas S. McGregor

December 12, 2007

SMART Laboratory

Department of Mechanical and Nuclear Engineering

Kansas State University

3018 Rathbone Hall

Manhattan, Kansas 66506

DOE/NNSA award DE-FG07-06ID14725 


\section{MATERIALS DEVELOPMENT}

\section{$\frac{\text { Purification }}{\text { A significa }}$}

A significant amount of progress has been achieved in the development of the novel vacuum distillation method described in the proposal. The process for the purification of Te was fully developed and characterized in a series of trials. The purification effect was confirmed with GDMS sample analysis and indicates the process yields very high purity Te metal. Results of this initial process study have been submitted for publication in the Proceedings of the SPIE and will be presented on August 28, 2007 at the SPIE Optics and Photonics 2007 conference in San Diego, CA.

Concurrent to the development of the Te process, processes for the purification of $\mathrm{Cd}, \mathrm{Zn}$, and $\mathrm{Mn}$ have also progressed. The development of the processes for $\mathrm{Cd}$ and $\mathrm{Zn}$ are nearly complete, while the development of the process for $\mathrm{Mn}$ is still in its infancy. It is expected that a full characterization of the $\mathrm{Cd}$ process will be completed within the next quarter, followed by $\mathrm{Zn}$. Parallel to those characterization studies, efforts will be made to further develop the $\mathrm{Mn}$ purification process.

Zone melting work for $\mathrm{Te}$ and $\mathrm{Cd}$ has also been efforted as per the project work schedule. Initial trials have been completed and the processes developed. Characterization of the results will be completed within the first fiscal year. Finally, an apparatus for the zone refinement of $\mathrm{Cd}$, $\mathrm{Zn}$, and Mn has been constructed and initial trials are set to begin shortly.

\section{Vacuum Distillation}

Vacuum distillation purification techniques rely on differences in the vapor pressures of the material of interest (in this case, tellurium) and the impurities present. When the temperature of a material is raised, the vapor pressure of the material also rises. By controlling the system temperature during evaporation, vacuum distillation can be used to harvest material with a desired vapor pressure.

Heating tellurium to above $452^{\circ} \mathrm{C}$ at atmospheric pressure would result in vaporization. However, the presence of oxygen and other impurities in the atmosphere would result in undesirable reactions likely to add further impurities to tellurium. By evacuating the source chamber, these impurities are removed. Additionally, the melting point is lowered, allowing higher vaporization rates at a lower temperature.

Selection of vaporization temperature is critical to successful purification. A temperature too low results in slow vaporization, which in turn cause slow condensate deposition. The corresponding purification rate is slow. However, higher temperatures will cause impurities with lower vapor pressures (relative to tellurium) to vaporize and condense on the deposition surface, minimizing the purification effect.

The vapor pressures of elements at $525^{\circ} \mathrm{C}$ are shown in Fig. 1. Vapor pressures for all elements except tellurium and selenium were calculated using data and equations from Dushman. The tellurium vapor pressure was calculated using equations from Machol and Westrum, and the selenium vapor pressure was obtained from Honig and Kramer. Some common impurity elements with higher vapor pressures include $\mathrm{Na}, \mathrm{S}, \mathrm{K}, \mathrm{Zn}, \mathrm{Se}$, and $\mathrm{Cd}$. Impurity elements with lower vapor pressures include $\mathrm{Mg}, \mathrm{Al}, \mathrm{Si}, \mathrm{Ca}, \mathrm{Fe}, \mathrm{Cu}, \mathrm{Ag}$, In, $\mathrm{Ba}$, $\mathrm{La}$, and $\mathrm{Pb}$. 


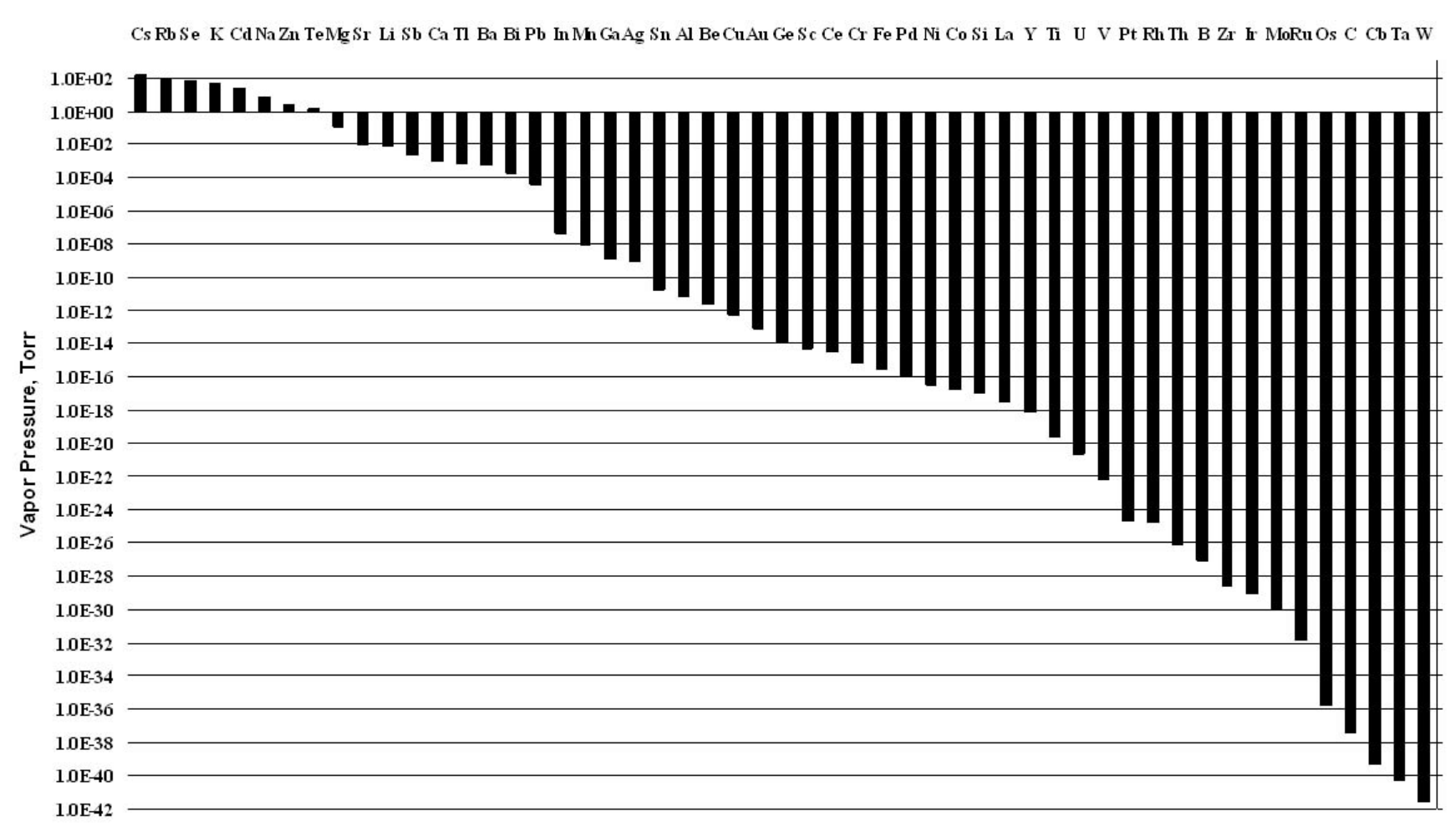

Fig. 1: Vapor pressures of common elements at $525^{\circ} \mathrm{C}$.

\section{Factors affecting purification}

In a vacuum distillation process, two phases are of particular importance for purification. One is the evaporation phase, and one is the condensation phase. Parameters can be controlled in both phases to control the purification process. In the evaporation phase, evaporation rate is a significant factor in the rate of material transfer. Vaporization rate is controlled by temperature, pressure, amount of surface area where vaporization can occur, composition of the material on the surface, and for impurities, the impurity concentration present in the raw material. Once vaporized, a material's mean free path as well as the temperature and material of the substrate determine whether the particle will condense in solid form, in liquid form, or will remain in the vapor state.

\section{Experimental Equipment}

A schematic representation of the basic experimental apparatus is shown in Fig. 2. The system consists of two subsystems: the vacuum system and the furnace system. The vacuum system includes a stationary, single use quartz ampoule coupled to a permanent vacuum system. A turbo pump coupled with a liquid nitrogen (LN2) trap can achieve ultimate vacuums of approximately $10^{-7}$ torr on an empty ampoule, as measured by a cold cathode ion gauge transducer.

The furnace system consists of a single zone resistance furnace with temperature and motion controls. A PID temperature controller provides temperature control with $+/-0.1^{\circ} \mathrm{C}$ precision. The heated zone is 12 inches in length with an internal diameter of 3 inches. Furnace motion is controlled using a motor coupled to a lead screw. The motion of the lead screw causes a counterweight to move up or down as required. The counterweight transfers the vertical motion to the furnace via a wire and pulley system. A National Instruments Labview program is 
used to control furnace motion and to continuously record temperature, pressure, and furnace position. An acid hood located in a Class 1000 clean room was used for ampoule cleaning and an inert atmosphere (nitrogen) glove box (dedicated to handling $\mathrm{Cd}, \mathrm{Zn}$, and Te) is used to load raw materials into the ampoule.

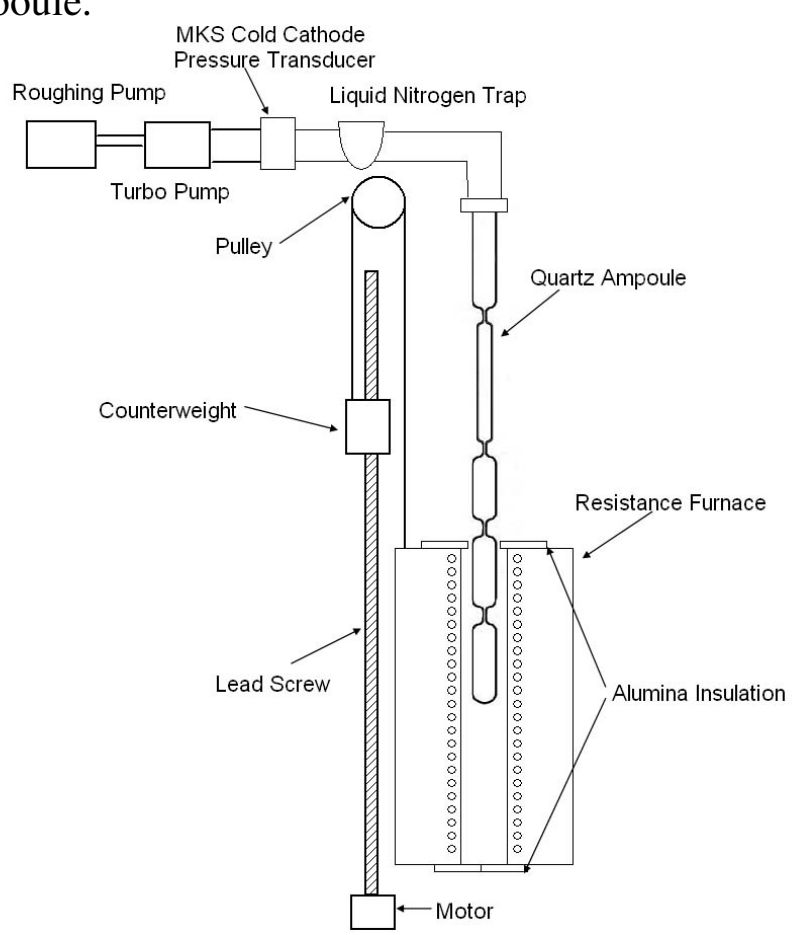

Fig. 2. Schematic drawing of tellurium distillation system.

\section{Experimental Procedure}

The quartz ampoule is cleaned in the Class 1000 clean room. A 1:1:1 $\mathrm{H}_{2} \mathrm{SO}_{4}: \mathrm{H}_{2} \mathrm{O}_{2}$ (35\%):DI $\mathrm{H}_{2} \mathrm{O}$ solution (piranha solution) is used as an etchant to remove organic impurities. This is followed by a $5 \%$ HF etch to remove a layer of the quartz, including any metallic impurities present. After thoroughly rinsing with deionized water, the ampoule is rinsed with isopropanol and allowed to dry in the clean room.

The cleaned ampoule is coupled to the glove box via a modified quick connect. This preserves the inert atmosphere of the glove box but allows loading a large ampoule using a small glove box. Tellurium raw material is measured and loaded through the top of the ampoule. The tellurium pieces drop down to the bottom chamber, now serving as the evaporation chamber. Once loaded, the ampoule is transferred to the vacuum system. A vacuum is established and allowed to stabilize. Typical working vacuums for the tellurium distillation are $10^{-6}$ torr.

Once the vacuum has stabilized, the furnace is heated to $200^{\circ} \mathrm{C}$ for a low temperature initial pass. The furnace is raised slowly over the length of the ampoule. This initial low temperature pass allows high vapor pressure impurities, such as water or solvents to evaporate and be drawn from the chamber by the vacuum system. After the initial 30-45 minute low temperature pass, the furnace is returned to a zero position, where the bottom of the ampoule is aligned with the top insulation surface of the furnace.

With the furnace at the zero position, temperature is raised to the distillation temperature of $525^{\circ} \mathrm{C}$ and allowed to stabilize. The furnace is then raised to the distillation start position, with the curved top surface of chamber B aligned with the top insulation on the furnace. The furnace is lowered slowly over the area of the distillation chamber. As the furnace lowers, a 
region on the ampoule is at the optimal temperature for tellurium condensation. Tellurium deposits on the chamber walls in an annular formation. At the end of the distillation run, temperature is lowered to ambient and the furnace is slowly lowered to below the zero position.

With the ampoule still evacuated, a hydrogen-oxygen torch is used to seal the neck between the evaporation chamber and the condensation chamber. The ampoule is now ready for the next stage of distillation. If this is the final distillation stage, the neck above the condensation chamber is also sealed.

Experimental results are presented for one, two, and three stage distillations of starting materials obtained from American Elements. The distillation processes labeled Te-VD-9, TeVD-10, and Te-VD-11 are from 4N nominal material obtained from American Elements. The single stage distillation (Te-VD-9) was done in a 3 chamber quartz ampoule while the two and three stage distillations were done in 5 chamber ampoules.

Approximate yields for each stage of distillation were calculated from the amount of residual material left after each stage, assuming that any material not left as residue is collected as purified material in the next chamber. This assumption overstates the yield due to loss of high vapor pressure impurities and some tellurium vapor to the vacuum system. The overall yield is calculated from the amount of purified material recovered compared to the amount of raw material initially loaded.

In calculating material purity, elements which were below the detectable limit were assumed to be equal to the limit of detection. For an estimate on the purity levels for the material where only partial GDMS analysis was conducted, the elements not tested for are assumed to be at the detection limit. The 25 elements selected to test for were the elements most likely to be present as impurities based on the initial material analysis provided by American Elements, those most likely to be introduced by the experimental process, and those most commonly detected by other researchers ${ }^{7,12-15}$. Although certified as $4 \mathrm{~N}+$ by American Elements, the sample from the initial raw material had $8.656 \mathrm{ppm}$ impurities, giving a material purity level of $99.99913 \%(5 \mathrm{~N})$. After a single stage of distillation (Te-VD-9) the impurity level dropped to $1.03 \mathrm{ppm}$ (99.999897\% pure). The two stage distillation (Te-VD-10) is $99.999843 \%$ pure. The three stage distillation (Te-VD-11) contained $0.92 \mathrm{ppm}$ total impurities, giving a material purity of 99.999908\% (6N). The largest appreciable source of impurities remaining in all three American Elements samples is selenium.

Selenium has a higher vapor pressure than tellurium so it would be expected to evaporate prior to tellurium. However, the optimal condensation temperatures of these materials are similar $^{12}$, thus minimizing the ability to remove selenium from the system. However, selenium removal can be done by bubbling hydrogen through molten tellurium, thus forming selenium hydride, which is removed with the hydrogen.

Purification of materials by vacuum distillation has been successful in the past year, particularly with tellurium. Tellurium raw materials have been purified to greater than $6 \mathrm{~N}$ purity (by full GDMS testing). One, two, and three stage distillation results were tested. The three stage purification results had less than $6 \mathrm{~N}$ impurities, with both the one and two stage impurity levels at better than $5 \mathrm{~N}$. (Starting material was nominally $4 \mathrm{~N}$ pure.) Average material yield varies between $75-85 \%$ of the initial charge per stage, depending on the initial impurity level of the material. The results were presented as a poster at SPIE Optics and Photonics 2007, titled "Purification of tellurium to $6 \mathrm{~N}$ using a multistage vacuum distillation method." Additional purification and testing of $3 \mathrm{~N}$ tellurium is in progress to help further characterize the capabilities of the multistage system. 


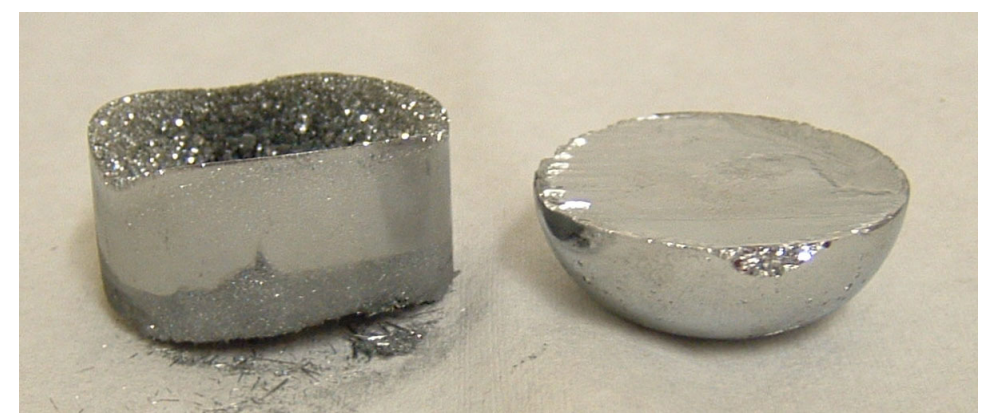

Fig. 3. Purified tellurium sample.

Zinc has also been successfully purified with vacuum distillation. $4 \mathrm{~N}$ raw material was purified to $5 \mathrm{~N}+$ in a three stage distillation process. The quartz ampoule design has been modified to increase the neck, allowing easier loading (and also lowering the ampoule cost!).

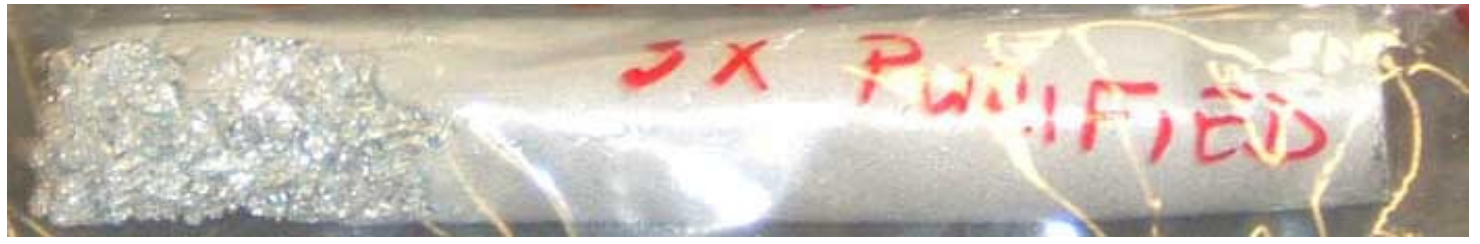

Fig. 4. Zinc sample, after 3 stages of purification.

Cadmium and manganese initial distillations have also been completed. The cadmium process is almost mature enough for material to be tested. Although cadmium does not react with quartz at distillation temperature, it does react at the ampoule sealing temperature. Thus multistage distillations require carbon coated ampoules. The manganese also requires carbon coated ampoules as it reacted detrimentally with the uncoated quartz on the initial run.

The normal freeze, or vertical zone melting method, of purification has also been attempted with cadmium, zinc and tellurium. Purified samples of tellurium have been analyzed as $6 \mathrm{~N}$ pure in the central portion of the sample, corresponding well with expected results. Cadmium and zinc samples are not yet ready to test.

\section{CdZnTe Growth}

The reaction of the constituent elements of CdZnTe to form the necessary compounds, CdTe and ZnTe, is a critical step towards the production of spectroscopic grade CdZnTe crystals. To this end, a full retrofit of a high pressure Bridgman furnace to serve as a reaction chamber is underway. First, the furnace was mounted and erected inside the SMART Laboratory. A new pressurization system that controls the chamber pressure from $1 \times 10^{-3}$ Torr $\left(1.9 \times 10^{-5}\right.$ psia) up to 100 atm (1470 psia) was installed and tested for operation. A redesign of the internal graphite structure as well as the heating elements is completed and awaiting installation. The temperature control system and power supply components were specified and are awaiting installation as well. The entire furnace system is expected to come online within the next quarter to begin reacting the purified elements produced by the vacuum distillation procedures being developed.

Growth of CdZnTe crystals from the melt has seen only modest progress. The switch from "counter-grade" $5 \mathrm{~N}$ purity $\mathrm{CdZnTe}$ raw material to higher grade $7 \mathrm{~N}$ pure raw material is 
underway. Unfortunately, furnace element failures have slowed progress. These issues have now been resolved by reconfiguring the furnace to operate at 120VAC as opposed to the previously used 240VAC to extend element life. Growth processes will resume by September 1, 2007.

Acquisition, installation and testing of a $50 \mathrm{~W} \mathrm{CO}$ laser system and an annealing furnace have been completed. Further, annealing effect metrics have been developed and an initial sample set prepared for annealing. Baseline thermal annealing studies are underway and will be followed by the introduction of the laser to the process. Finally, a thermal model of the annealing process is under development and showing promising results. Initial laser annealing results are expected slightly ahead of schedule.

\section{CdZnTe Annealing}

Cadmium zinc telluride $(\mathrm{CdZnTe})$ detectors have demonstrated that room-temperature energy resolutions less than $1.5 \%$ for $662 \mathrm{keV}$ gamma rays can be achieved. Tellurium (Te) precipitates however plague device performance in CdZnTe spectrometers. The migration of Te precipitates due to an externally applied thermal gradient has been proven. The CdZnTe preferentially dissolves at the hotter edge of the precipitate and tends to recrystallize at the cooler edge thereby moving the precipitate toward the hotter region. The premise of the present investigation is to use a light wavelength that readily transmits through the CdZnTe but is sufficiently absorbed by Te precipitates to induce a temperature gradient in the precipitate.

Finite difference thermal simulations were performed to set the parameters for first experimental runs. The simulations suggest that the energy deposited on the precipitates during milliseconds may provide a sufficient thermal gradient. The steep gradient leads to increased conduction such that longer laser exposure does not increase the gradient but heats up the entire crystal. Intermittent laser power leads to rapid local cooling which prevents local crystal melting.

In initial laser irradiation trials, heat loss due to natural convection prevented the bulk crystal from reaching appropriate annealing temperatures. The experimental setup was then modified such that the crystal was placed in a single zone furnace which provided supplementary heat and a constant thermal environment. Initial results exhibited a darkened crystal after an extensive combined laser-heat annealing run which may be due to Te outdiffusion. This circumstance made the identification of precipitates harder and no clear precipitate movement could be observed. Based on this, further experimental runs with lowered background temperature and optimized laser pulse timing will be conducted.

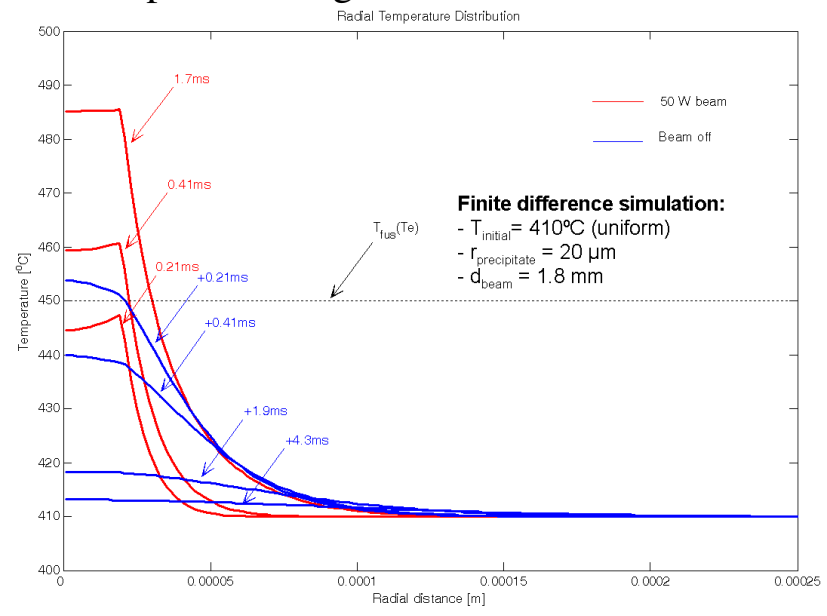

Fig. 5: Finite difference thermal simulation. Laser beam energy is simulated to be deposited at precipitate surface. 


\section{$\underline{\text { LaBr}_{3}} \underline{\text { Growth }}$}

Growth of $\mathrm{LaBr}_{3}: \mathrm{Ce}$ crystals from the melt has progressed well. The growth process has seen several refinements in the past nine months as four crystal growth runs were completed. As a result, the average size of the single crystals within the grown ingot is increasing. Future planned furnace modifications are expected to greatly improve thermal environment control and, therefore, improve the consistency of results and increase the average size of single crystals produced. Study of the distribution of activator dopants will commence on schedule in FY08.

Recently, several new alloys of $\mathrm{CeBr}_{3}$ were developed at Sandia National Laboratories with funding from NNSA in a joint effort with a graduate research assistant, Mark Harrison, from the SMART Laboratories at KSU to improve the strength of the available lanthanide halide scintillator materials. These new alloys are expected to have critical resolved shear stresses (CRSS) an order of magnitude larger than the base compounds. The alloying work was a direct response to the fragility of the lanthanide halides and the associated difficulties in growing large single crystals. In an informal partnership with SNL, the SMART Laboratories at KSU will be growing ingots of these new alloys to verify the improved ease of crystal growth. Confirmation of strengthening effects will be an exciting achievement for the science and an outstanding benefit to the gamma-ray spectroscopy community.

Much progress has been made toward producing and characterizing large, single crystals of lanthanide halides and their alloys. Three key areas have seen progress, including crystal growth, sample preparation, and characterization capabilities. Crystal growth, in particular, has experienced the greatest advances.

The vertical Bridgman crystal growth system was reprogrammed to enable greater process monitoring capabilities and has since been re-characterized and tested. It is currently growing a $150 \mathrm{~g}$ charge of $\mathrm{LaBr}_{3}$ doped with $5 \% \mathrm{CeBr}_{3}$. The horizontal Bridgman technique was investigated with a horizontal electro-dynamic gradient (EDG) furnace as a lower-stress method for growing lanthanide halide single crystals as compared to vertical Bridgman or Czochralski methods. Initial results, shown in Fig. 6, with a $150 \mathrm{~g} \mathrm{LaBr}_{3}$ charge exhibited large, crack-free single crystals. Due to the encouraging results, a second horizontal EDG has been committed to further exploring this method of growth. Both horizontal EDG furnaces are currently engaged in crystal growth runs.

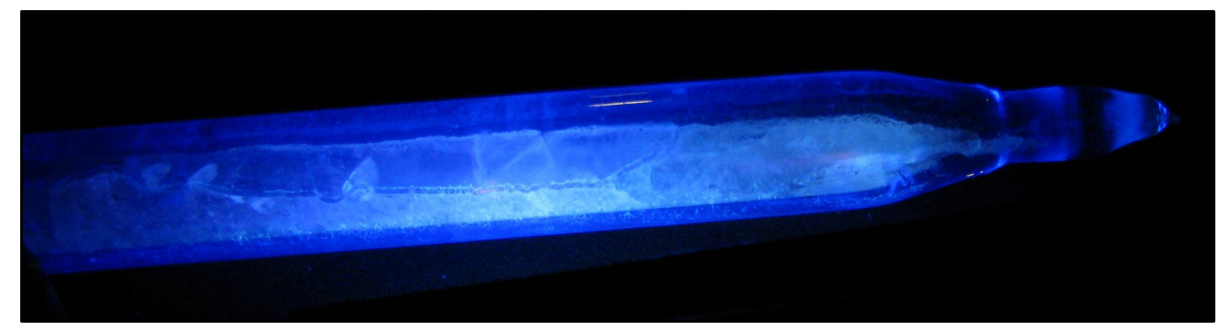

Fig. 6. First 5\% Cerium-doped $\mathrm{LaBr}_{3}$ ingot grown via the horizontal Bridgman technique.

Sample preparation capabilities have also been achieved with the completion of the refurbishment of a diamond-wire saw. Crystal cutting, grinding and polishing are all now possible completely within ultra-dry, inert atmospheres. The ability to mount and test samples has also been achieved. Spectra with $8.3 \%$ FWHM at $662 \mathrm{keV}$ energy resolution have been 
recorded as shown in Fig. 7. Energy resolution is expected to improve when better photomultiplier tubes have been acquired. A spectrophotometer has recently been acquired to measure the radioluminescence spectra of the new alloys. Finally, a Compton coincidence light proportionality measurement system has been brought on line and is undergoing characterization.

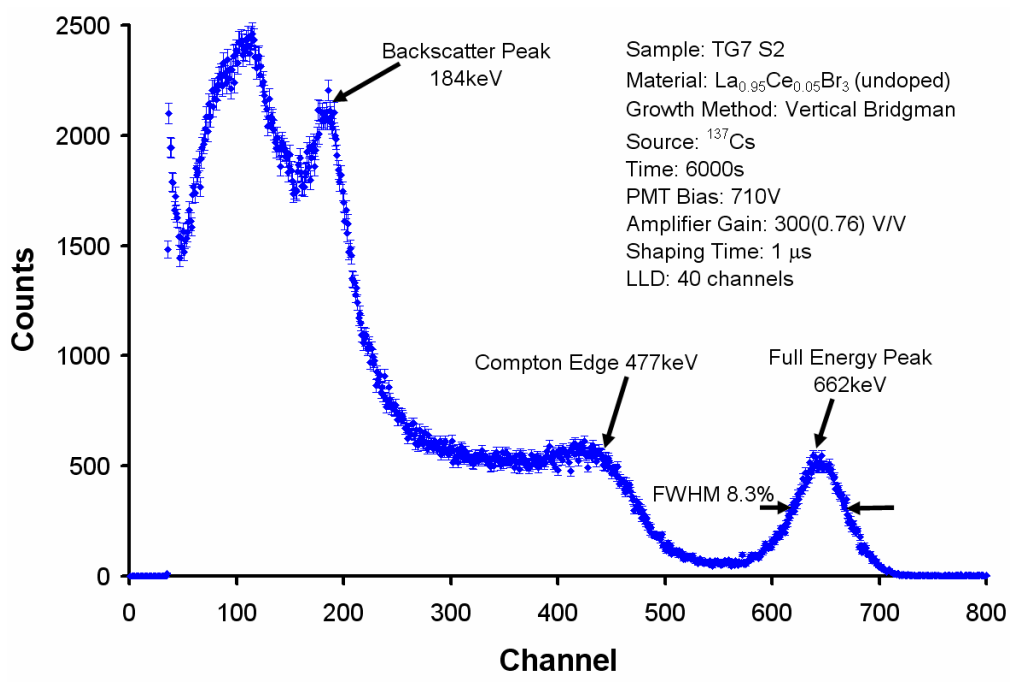

Fig. 7. Pulse height spectrum recorded with vertical Bridgman grown cerium-doped $\mathrm{LaBr}_{3}$.

\section{Horizontal Furnaces/Mercuric Iodide (HoI 2 ) Platelet Growth}

SMART Laboratory has forty horizontal furnaces for mercuric iodide $\left(\mathrm{HgI}_{2}\right)$ platelet growth experiments (thirty one two-zone furnaces and nine one-zone furnaces). The temperature of thirty furnaces (twenty one two-zone furnaces and all one-zone furnaces) is controlled with the ADAPT system (produced by the Mellen Company Inc.), which was installed in June 2006. Temperature fluctuation of no more than $+/-0.2^{\circ} \mathrm{C}$ is required for better growth control, however, the ADAPT system could not achieve this condition with the two-zone furnaces prior to February 2007. Lowering the power supplied to the furnace zones was determined as the solution, hence two transformers were purchased and installed in March 2007. Furnace rewiring was completed in May 2007. Current average temperature fluctuation has been recorded to be less than $+/-0.2^{\circ} \mathrm{C}$. The temperature profile of the aforementioned thirty furnaces has been collected since May 2007.

Platelet growth experiments were started in the first week of August 2007. The purpose of these experiments is to determine factors affecting platelet growth in horizontal furnaces. The first set of experiments utilizes polyethylene powder as additive in platelet growth. The method of factorial analysis will be used to analyze the length of the growth zone as well as the size and yield of the platelets. Current set of experiments will investigate the effects of hot zone temperature, temperature gradient and amount of polyethylene powder in platelet growth. The rest of the experimental sets will utilize high purity gases instead of polyethylene addition. The gases to be used are high purity $\mathrm{N}_{2}, \mathrm{Ar}, \mathrm{O}_{2}$, and $\mathrm{H}_{2}$. The factorial analysis method will also be utilized to determine the corresponding effects. 


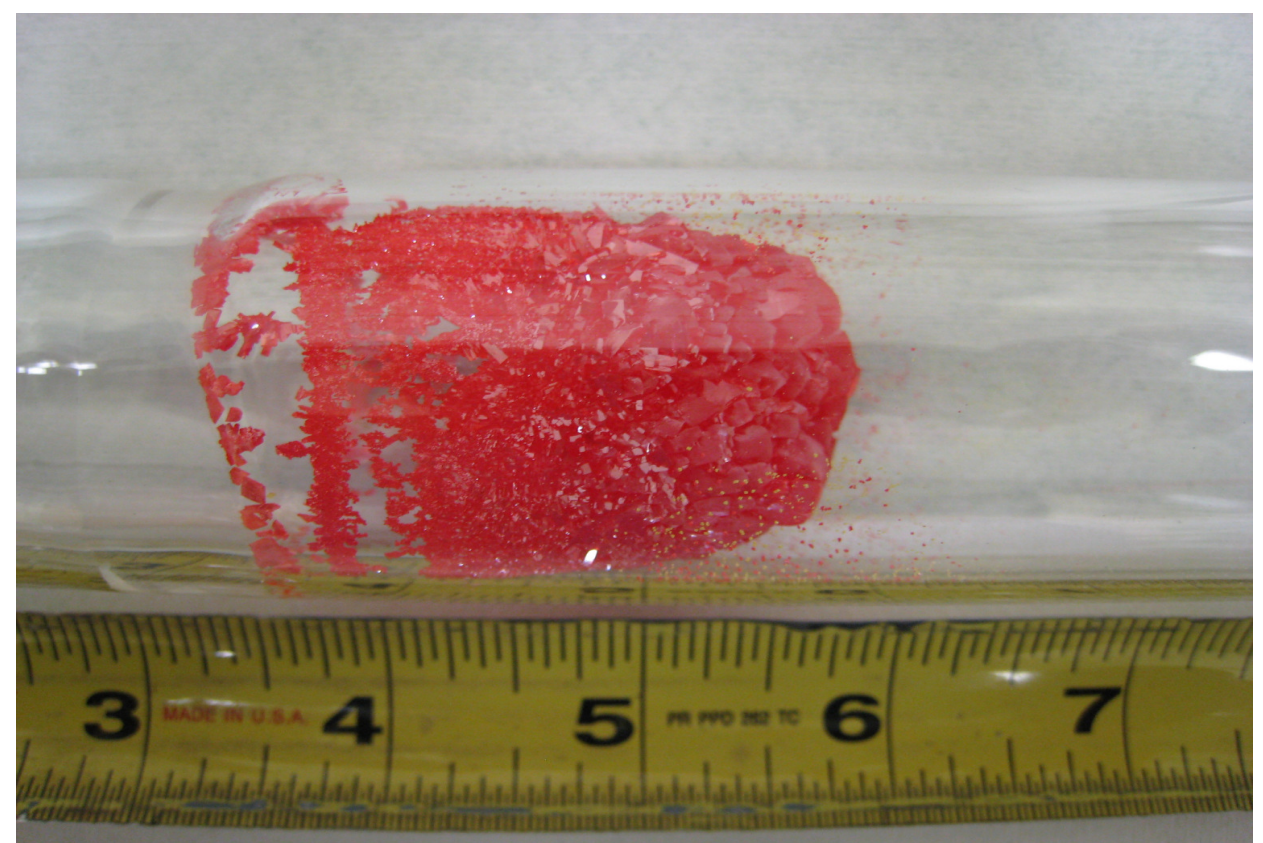

Fig. 8: $\mathrm{HgI}_{2}$, after a polyethylene addition process, ready to be retrieved and used as a growth source.

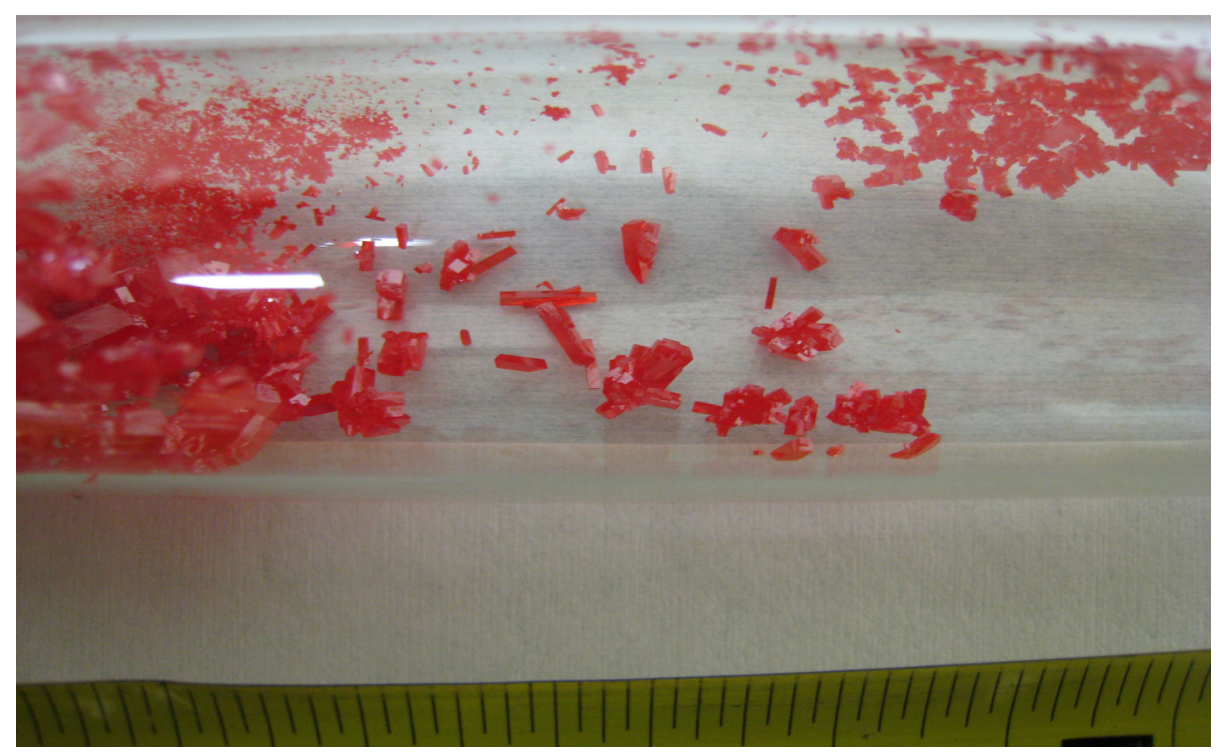

Fig. 9: One of the preliminary growth runs with polyethylene addition.

\section{GAMMA RAY DETECTOR DEVELOPMENT}

\section{Mercuric Iodide Detector Development}

A solution string saw currently utilizing potassium iodide (KI) solution and Kevlar thread as string was completed in June 2007. The saw has been employed to cut $\mathrm{HgI}_{2}$ crystals to size. Planar detectors have been fabricated with crystals cut to 5 x $5 \mathrm{~mm}^{2}$ surface area and thickness ranges from $0.4 \mathrm{~mm}$ to $1 \mathrm{~mm}$. Cleaved crystals are polished and etched with KI solution. After being rinsed in DI- $\mathrm{H}_{2} \mathrm{O}$, the crystals are subsequently dried with $\mathrm{N}_{2}$ gun. Currently Aquadag paint is applied as contact material and palladium wires as conducting wires. Sputtered palladium and platinum will also be used as contact materials. 
Protective coating of $\mathrm{HgI}_{2}$ detectors has been shown to preserve detector stability over time. Beginning July 2007 Teflon AF (made by Dupont) used as is or diluted with FC-72 (made by $3 \mathrm{M}$ ) has been employed to coat several fabricated detectors. Results after 6 hours of applied bias show little change in ${ }^{241} \mathrm{Am}$ spectra of detectors coated with diluted Teflon AF. Parylene C and $\mathrm{N}$ films will also used as protective coating.

Methods to fabricate spectrometer-quality planar detectors will be optimized. Planar detectors fabricated with Aquadag contacts does not perform satisfactorily as spectrometers, with an average FWHM of $15 \%$ for ${ }^{241} \mathrm{Am}$ full energy peak at $59.6 \mathrm{keV}$. Various detectors designs including Frisch ring devices will be fabricated.

NIM equipment for detector testing were acquired in March 2007 and a system to simultaneously test ten $\mathrm{HgI}_{2}$ detectors was constructed and tested in June 2007. Each detector box has a detector stage AC-coupled to a CR-101D or CR-110 preamplifier (produced by Cremat Inc.). Detectors in ten boxes are biased simultaneously with a high voltage power supply through a junction box. The preamplifiers are connected to the DC power outputs of the NIM bin through voltage regulators that are also housed in the junction box. Connectors consist of BNC cables (puchased by June 2007) and SHV cables (fabricated by June 2007).

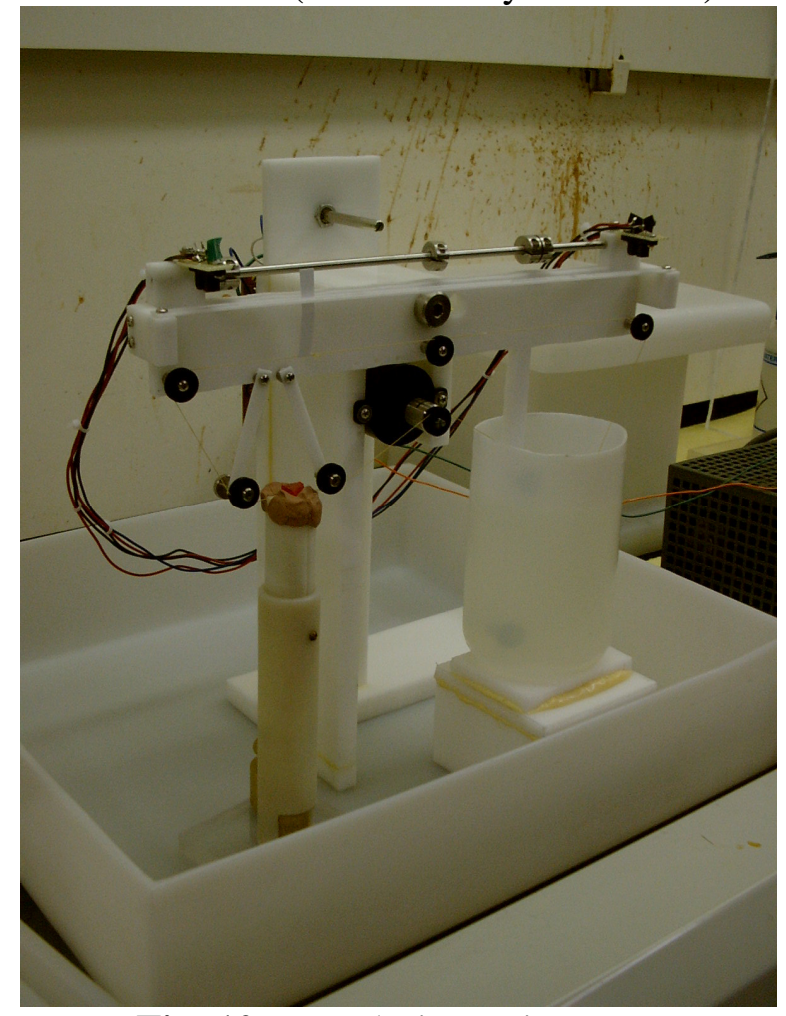

Fig. 10: KI solution string saw. 


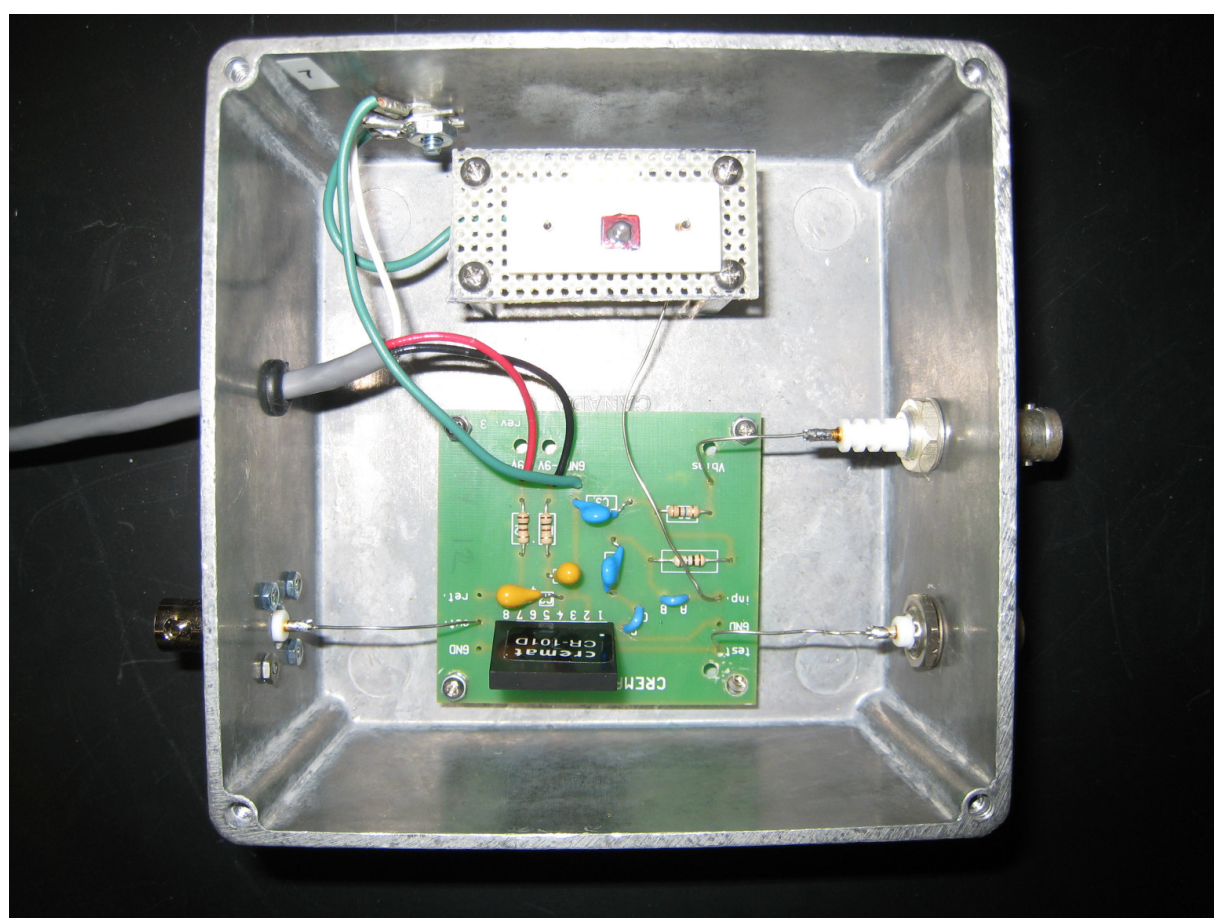

Fig. 11: Detector and preamplifier box.

\section{Accomplishments/New Findings}

Planar samples have been fabricated for crystal characterization. The quality of a $\mathrm{HgI}_{2}$ spectrometer depends on material quality, fabrication processes, and post-fabrication processes (encapsulation for detector stability in the long run), hence the performance of the spectrometer can be a method of characterization. A multi-detector testing has been built to accommodate testing ten detectors simultaneously, thus effective crystal characterization can be performed. The setup can be used not only to collect spectra, but eventually to measure charge carrier mobilities of grown crystals. Complete investigation of charge carrier mobilities will enable better designs of spectrometers made of single crystals of $\mathrm{HgI}_{2}$. 


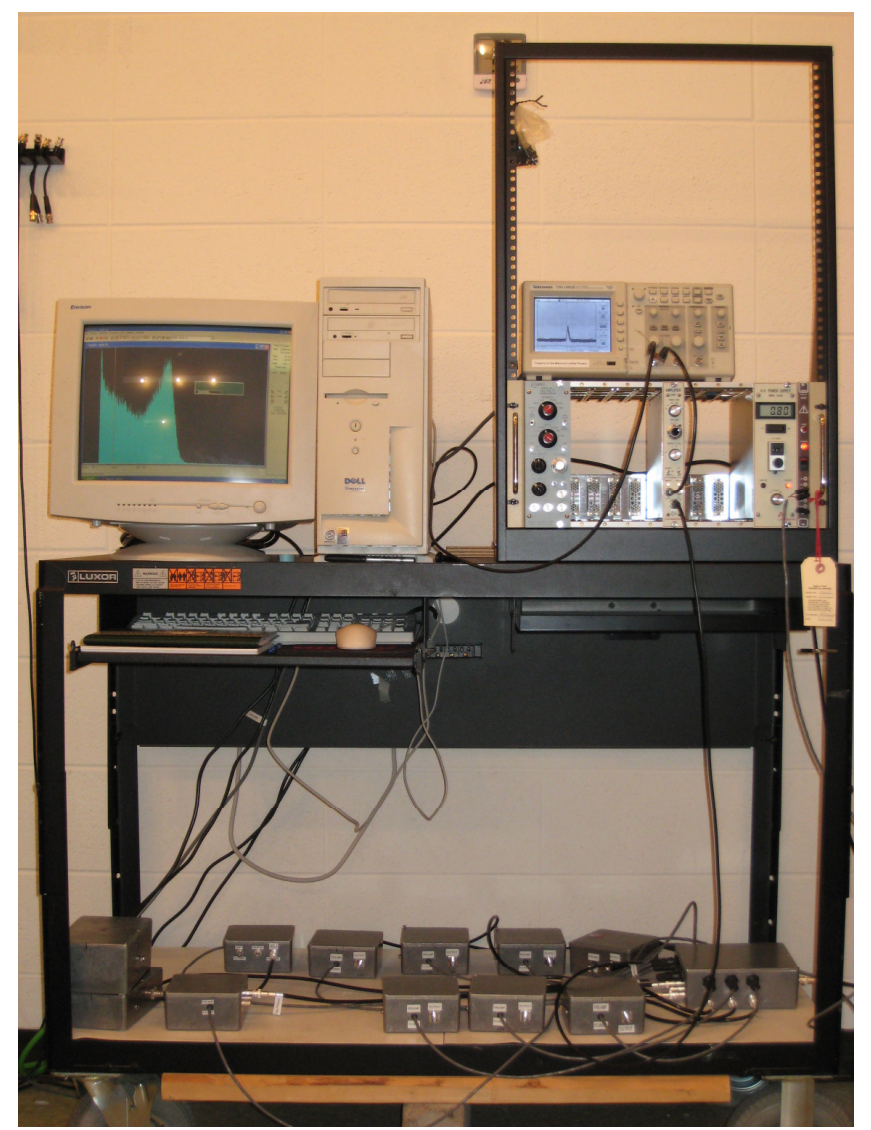

Fig. 12: Multi-detector system.

\section{CdZnTe Detector Development}

The objective of this project is to fabricate sixteen CdZnTe Frisch collar detectors. The CdZnTe detectors will have dimensions of $3 \times 3 \times 6 \mathrm{~mm}^{3}$ up to $4.5 \times 4.5 \times 9.5 \mathrm{~mm}^{3}$ with an energy resolution of about $2 \%$ at $662 \mathrm{keV}$. The CdZnTe detectors will be tested using a ${ }^{137} \mathrm{Cs}$ standard gamma ray source. The ultimate goal for this project is to investigate the feasibility of CdZnTe Frisch collar detectors for imaging applications. Hence, the schematic of the proposed arrangement for both collimated and non-collimated CdZnTe Frisch collar detectors are given in 6. As shown, the CdZnTe bar detectors are primarily wrapped (coated) with an insulator and then with a conductive collar. Later, the detectors are inserted into a collimator (collimated array), or mounted on a substrate to be used as a non-collimated array of CdZnTe devices. Further, a systematic investigation will be performed to study the effect of different dielectric materials as the insulator for the Frisch collar devices. This also involves studying different deposition methods for the insulator and Frisch ring. Later, the systematic investigation extends to study different methods of depositing the contacts on CdZnTe detectors and different methods of passivating the devices.

To achieve the objectives outlined above, many CdZnTe Frisch collar detectors of different sizes have been fabricated. Due to lack of high quality CdZnTe growth materials and difficulty in acquiring decent growth materials, previously acquired CdZnTe was used as the starting material. These materials were obtained from Redlen Technology, Digirad, Yinnel Tech, Inc., Washington State University, Bicron, and eV PRODUCTS. The CdZnTe Frisch collar detectors with the best energy resolution were fabricated from Redlen Technology growth 
materials (1.05\% at $662 \mathrm{keV}$ Shown on 3.22). It should be emphasized that there was about $80 \%$ yield for the fabrication process to make the CdZnTe Frisch collar detectors; also about 50\% yield for the material quality to maintain energy resolution of less than $2.5 \%$ at $662 \mathrm{keV}$.

To facilitate taking data from the array of CdZnTe detectors a Readout Electronics for Nuclear Application (RENA-3) was acquired. The RENA-3 is a 36-channel charge sensitive amplifier/shaper ASIC chip with trigger output and sparse readout mode. The RENA-3 is designed for reading out position sensitive solid state detectors, and is developed by NOVA R\&D, Inc. Figure 15 shows the RENA-3 package with the two mounted CdZnTe detectors, being tested simultaneously. The initial data from a ${ }^{137} \mathrm{Cs}$ check source was acquired with the two CdZnTe detectors using the RENA-3 package. A comparison of the spectra taken with standard NIM system/142A ORTEC preamplifier and the spectra taken with the RENA-3 package are shown in Fig. 16 for one of the tested detectors.

More recently, the effects of the crystal aspect ratio on spectral performance of CdZnTe Frisch collar devices were investigated by the PI. A $19.08 \times 19.34 \times 4.95 \mathrm{~mm}^{3}$ device fabricated from CdZnTe grown by Redlen Technologies was used as the starting material. The crystal was re-fabricated many times to achieve several aspect ratios while the device length was held constant at $4.8 \pm 0.2 \mathrm{~mm}$ (Figure 17). The following aspect ratios were successfully fabricated from the initial device: $0.26,0.52,0.71,0.96,1.19,1.36,1.59$ and 1.92 (see Table). The energy spectra of ${ }^{241} \mathrm{Am}$ and ${ }^{137} \mathrm{Cs}$ were recorded for all devices in both planar and Frisch collar configurations. The energy spectra of ${ }^{137} \mathrm{Cs}$ taken from all fabricated devices are presented in. It was observed that the Frisch collar effect begins to occur for devices with an aspect ratio of approximately 1.0. Device performance continued to improve as the aspect ratio was further increased and was noted to significantly improve the energy resolution of the device when the aspect ratio was greater than 1.5 . The CdZnTe devices were also theoretically modeled to support the experimental conclusion.

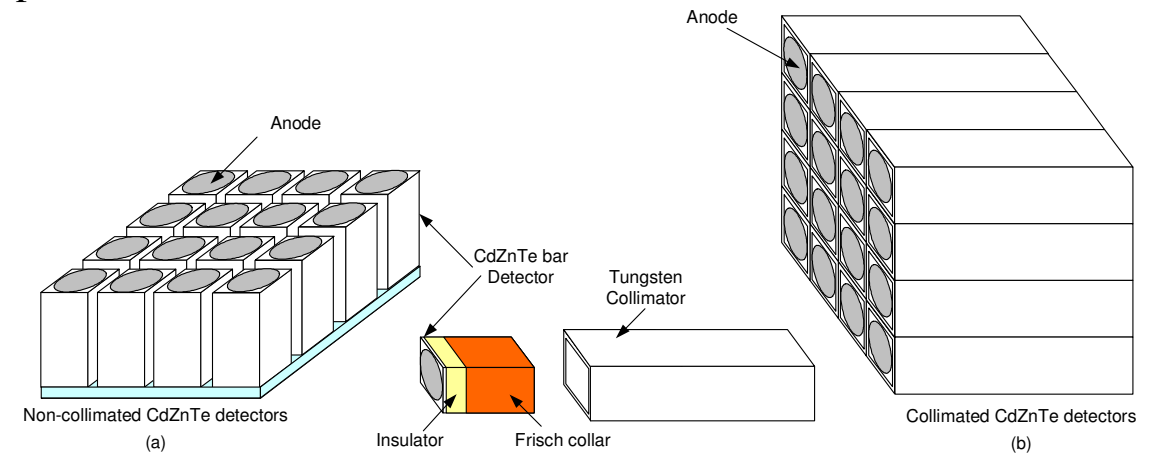

Fig. 13: The schematic of CdZnTe Frisch collar detectors (a) $4 \times 4$ array of non-collimated detectors (b) $4 \times 4$ array of collimated detectors. 


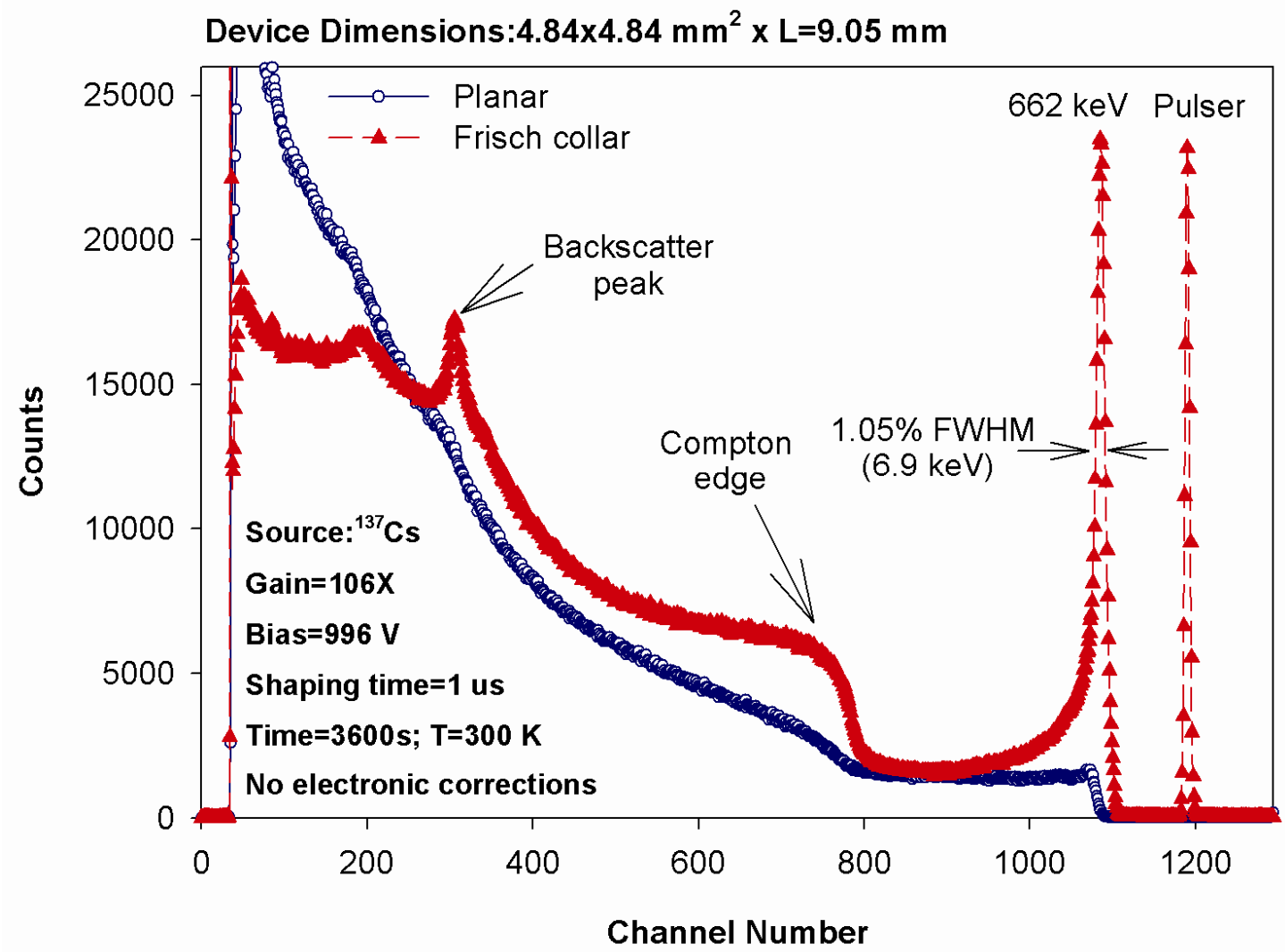

Fig. 14: The pulse height energy spectrum of ${ }^{137} \mathrm{Cs}$ for $3.59 \times 3.59 \times 8.19 \mathrm{~mm}^{3} \mathrm{CdZnTe}$ device fabricated from Redlen Technology materials.

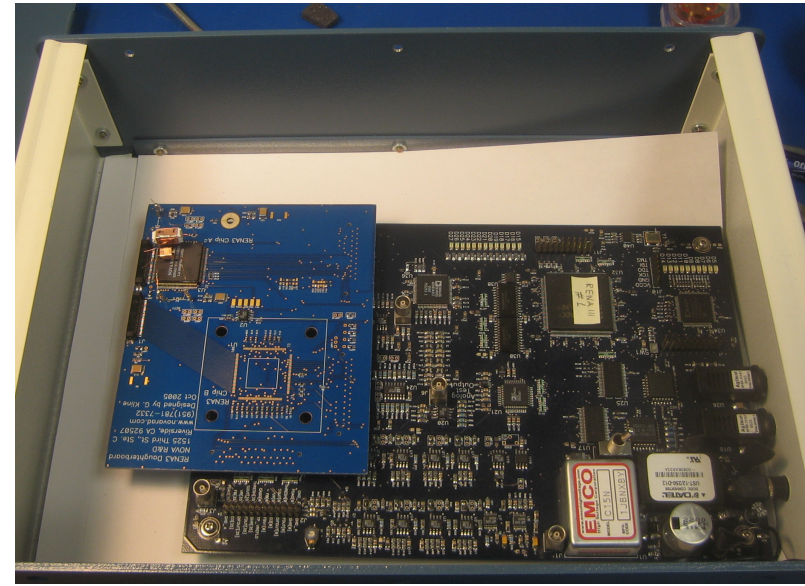

(a)

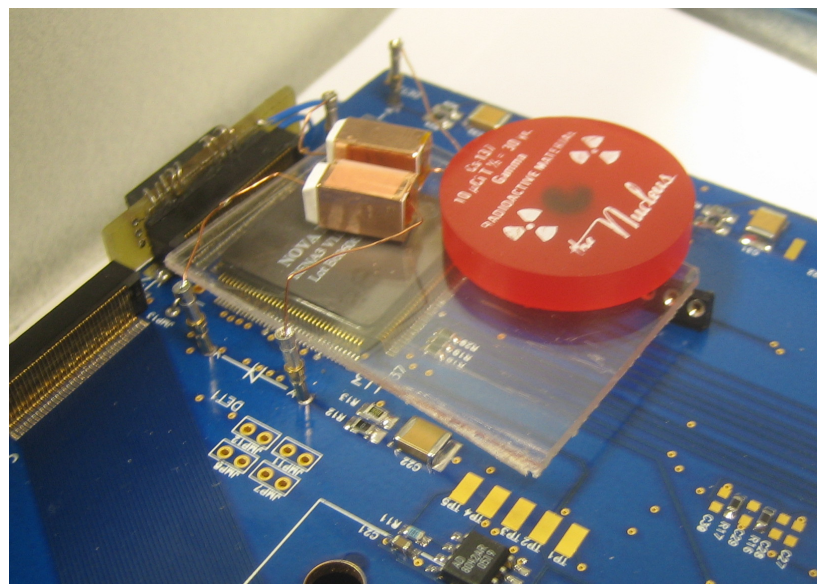

(b)

Fig. 15: The RENA3 chips package (a) The package mother board and daughter board with two CdZnTe Frisch collar detectors mounted for spectral test (b) The two CdZnTe non-collimated Frisch collar detectors mounted for spectral testing with Cs-137 simultaneously. 

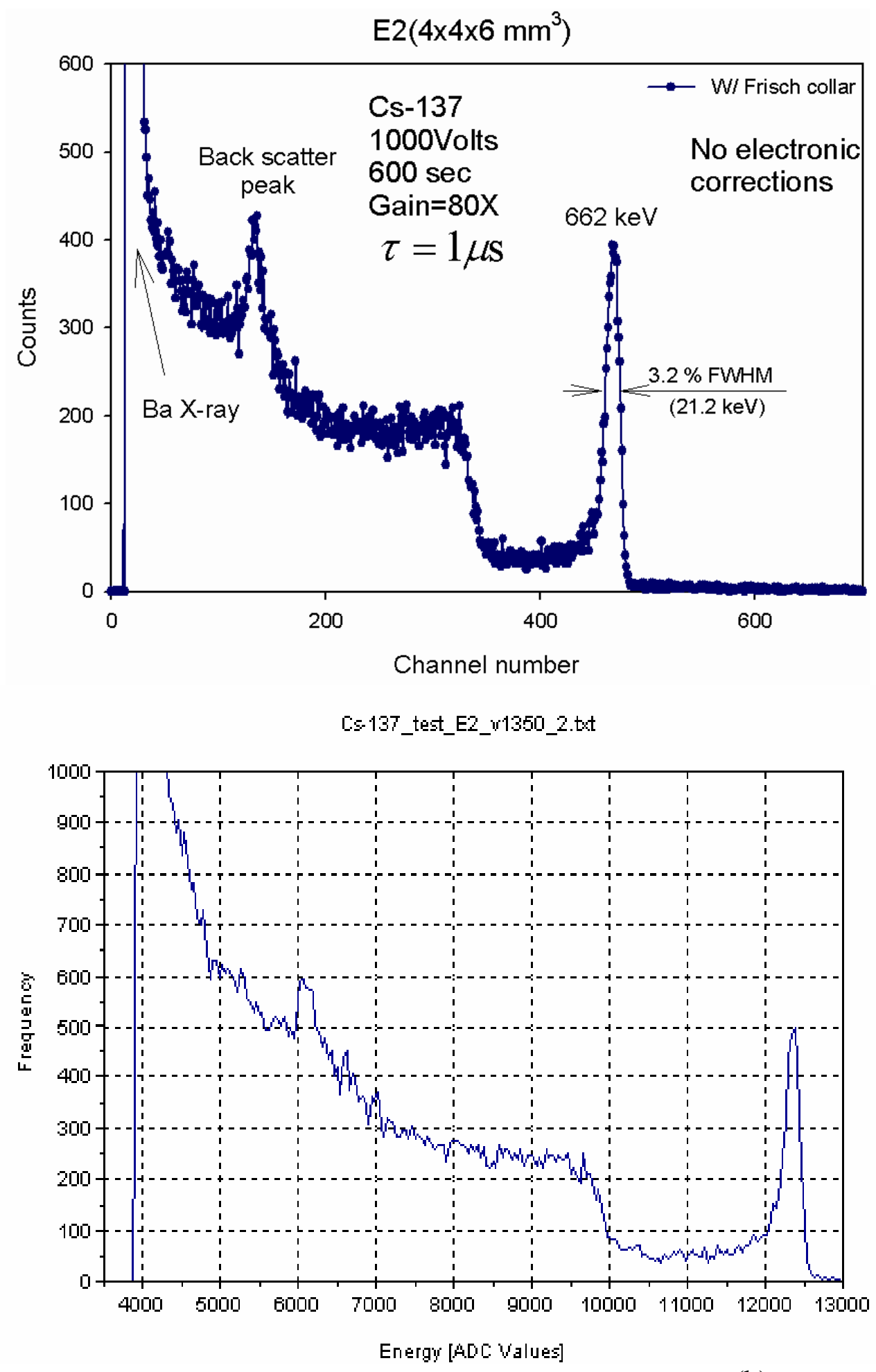

(b)

Fig. 16: The pulse height spectra with ${ }^{137} \mathrm{Cs}$ from the $4 \times 4 \mathrm{~mm}^{2} \times \mathrm{L}=6 \mathrm{~mm}$ CdZnTe Frisch collar detectors fabricated from Digirad growth materials (a) with standard NIM system and 142A preamplifier (b) the primary test with RENA3 chips package (note that the energy lines are not calibrated). 


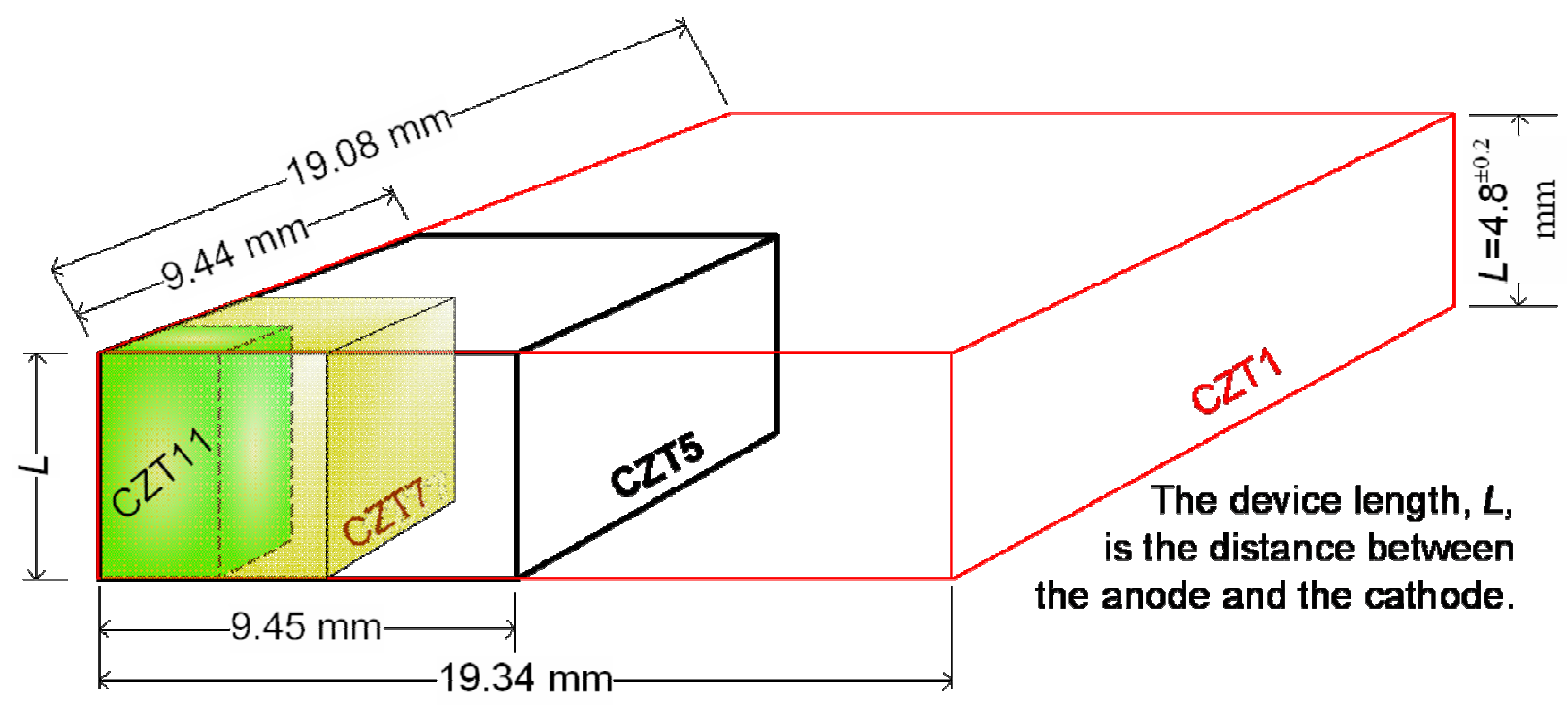

Fig. 17: Sample devices for each strategy showing the initial CZT1 (entire sample) and the some of the fabricated devices.

Table 1: The fabricated CdZnTe detectors name, dimensions and aspect ratio and the applied bias.

\begin{tabular}{|c|c|c|c|c|}
\hline $\begin{array}{l}\text { Detector } \\
\text { name }\end{array}$ & Dimensions (mm) & $\begin{array}{c}\text { Average } \\
\text { aspect ratio } \\
(L / W)\end{array}$ & $\begin{array}{l}\text { Applied } \\
\operatorname{bias}(V)\end{array}$ & Spectral testing time (s) \\
\hline CZT1 & $19.08 \times 19.34 \times L=4.95$ & 0.26 & 800 & $\begin{array}{c}\text { 1800s for }{ }^{137} \mathrm{Cs}, 900 \text { s for } \\
{ }^{241} \mathrm{Am}\end{array}$ \\
\hline CZT5 & $9.44 \times 9.45 \times \mathrm{L}=4.88$ & 0.52 & 800 & $\begin{array}{c}\text { 1800s for }{ }^{137} \mathrm{Cs}, 900 \text { s for } \\
{ }^{241} \mathrm{Am}\end{array}$ \\
\hline CZT6 & $6.65 \times 6.89 \times \mathrm{L}=4.84$ & 0.71 & 800 & $\begin{array}{c}\text { 3600s for }{ }^{137} \mathrm{Cs}, 1800 \mathrm{~s} \\
\text { for }{ }^{241} \mathrm{Am}\end{array}$ \\
\hline CZT7 & $5.05 \times 5.02 \times \mathrm{L}=4.84$ & 0.96 & 800 & $\begin{array}{c}\text { 5200s for }{ }^{137} \mathrm{Cs}, 2600 \mathrm{~s} \\
\text { for }{ }^{241} \mathrm{Am}\end{array}$ \\
\hline CZT8 & $4.03 \times 4.00 \times \mathrm{L}=4.76$ & 1.19 & 800 & $\begin{array}{c}\text { 7200s for }{ }^{137} \mathrm{Cs}, 3600 \mathrm{~s} \\
\text { for }{ }^{241} \mathrm{Am}\end{array}$ \\
\hline CZT9 & $3.51 \times 3.51 \times \mathrm{L}=4.76$ & 1.36 & 800 & $\begin{array}{c}9000 \text { s for }{ }^{137} \mathrm{Cs}, 4500 \mathrm{~s} \\
\text { for }{ }^{241} \mathrm{Am}\end{array}$ \\
\hline CZT10 & $2.90 \times 2.90 \times \mathrm{L}=4.60$ & 1.59 & 800 & $\begin{array}{c}10800 \text { for }{ }^{137} \mathrm{Cs}, 5400 \text { for } \\
{ }^{241} \mathrm{Am}\end{array}$ \\
\hline CZT11 & $2.40 \times 2.40 \times \mathrm{L}=4.60$ & 1.92 & 800 & $\begin{array}{c}12600 \text { for }{ }^{137} \mathrm{Cs}, 6300 \text { for } \\
{ }^{241} \mathrm{Am}\end{array}$ \\
\hline
\end{tabular}




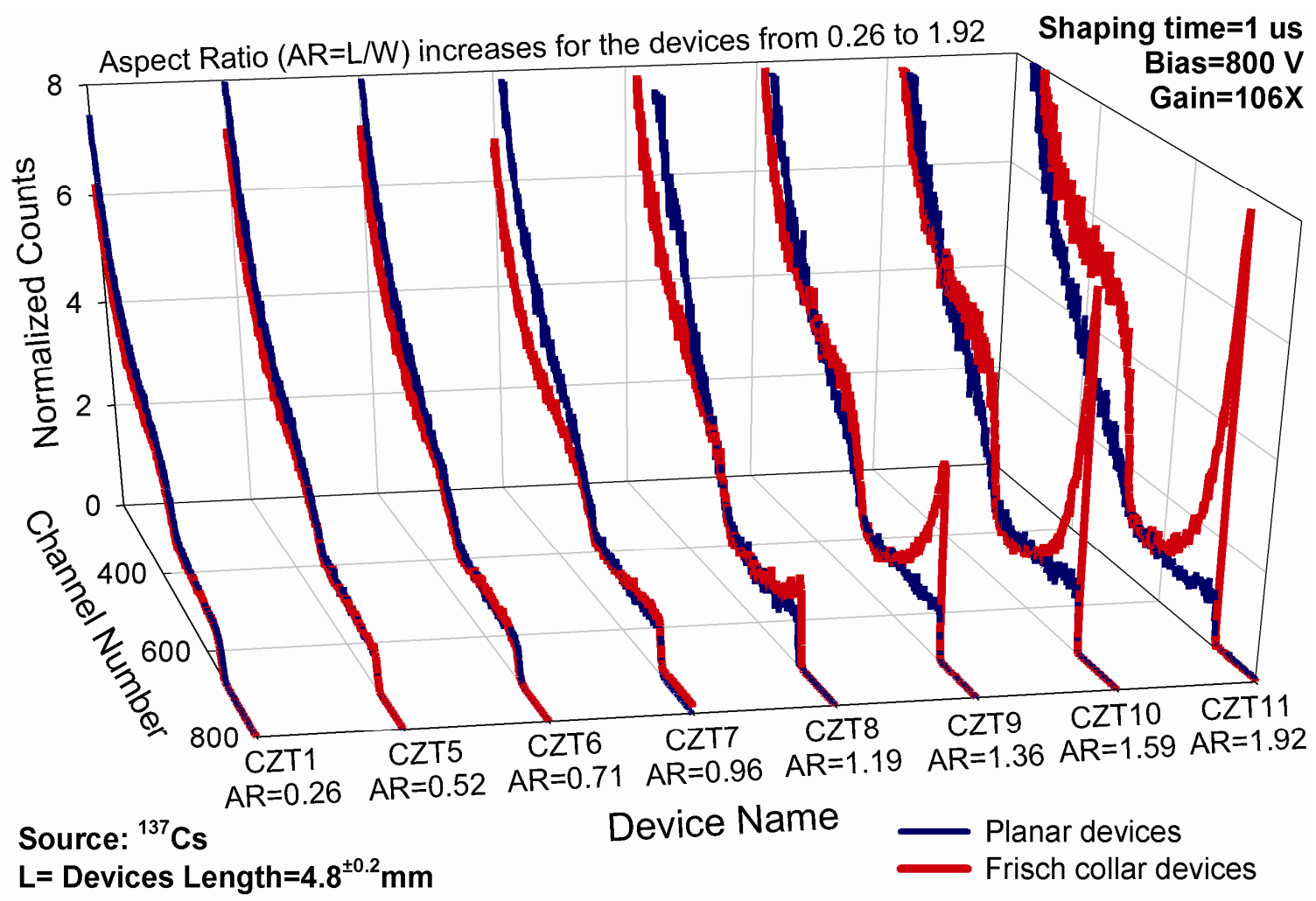

Fig. 18: The Normalized pulse height spectra collected from devices listed in Table 1 as: CZT1, CZT5, CZT6, CZT7, CZT8, CZ9, CZT10 and CZT11 in planar and Frisch collar configurations using a ${ }^{137} \mathrm{Cs}$ source. 


\section{PUBLICATIONS RESULTING IN PART FROM THE WORK}

1. M.J. Harrison, A. Kargar, D.S. McGregor, "Improved Fabrication of Frisch Collar CdZnTe Gamma Ray Spectrometers," IEEE Nuclear Science Symposium, Waikiki, Hawaii, Oct. 28Nov. 3, 2007.

2. Y. Cui, A. Bolotnikov, G. Camarda, A. Hossain, R.B. James, G. De Geronimo, J. Fried, P. O'Connor, A. Kargar, M. J. Harrison, D. S. McGregor, "Hand-Held Gamma-Ray Spectrometer Based on High-Efficiency Frisch-Ring CdZnTe Detectors," IEEE Nuclear Science Symposium, Waikiki, Hawaii, Oct. 28-Nov. 3, 2007.

3. F.P. Doty, D. McGregor, M. Harrison, K. Findley, R. Polichar, "Structure and Properties of Lanthanide Halides," Proc. SPIE, 6707 (2007) pp. 051-0511.

4. A.M. Hageman, M.J. Harrison, N. Fritz , T.N. Krehbiel, R. White, J. Patenaude, D.S. McGregor, "Purification of Tellurium to $6 \mathrm{~N}$ Using a Multistage Vacuum Distillation Method,” Proc. SPIE, 6707 (2007) pp. oZ1-oZ7.

5. A. Kargar, R.B. Lowell, M.J. Harrison and D.S. McGregor, "The Crystal Geometry and the Aspect Ratio Effects on Spectral Performance of CdZnTe Frisch Collar Device,” Proc. SPIE, 6706 (2007) pp. 1J1-1J17.

6. K.C. Mandal, S.H. Kang, M. Choi, A.Kargar, M.J. Harrison, D.S. McGregor, A.E. Bolotnikov, G.A. Carini, G.C. Camarda, R.B. James, "Characterization of Low Defect $\mathrm{Cd}_{0.9} \mathrm{Zn}_{0.1} \mathrm{Te}$ and CdTe Crystals for High Performance Frisch Collar Detectors," IEEE Trans. Nucl. Sci, (2007) in press.

7. M.J. Harrison, A. Kargar, D.S. McGregor, "Charge Collection Characteristics of Frisch Collar CdZnTe Gamma Ray Spectrometers," Nucl. Instrum. and Meth. A, A579 (2006) pp. 134-137.

8. Y. Cui, A. E. Bolotnikov, G. S. Camarda, G. De Geronimo, P. O'Connor, R. B. James, A. Kargar, M. J. Harrison, and D. S. McGregor, "Readout System for Arrays of Frisch-ring CdZnTe Detectors," IEEE Nuclear Science Symposium, San Diego, CA, Oct. 29-Nov. 3, 2006. 